isms contain both chlorophyll $a$ and $b$ as found in eukaryotic plant cells (see Nature, 261, 697; 1976). Could these organisms or relatives be ancestral forms of photosynthetic plastids of eukaryotic cells? Whether this type of "prokaryote" is a missing link or not remains to be seen, but it is likely to be a link between this and the third symposium.

\section{Anti-juvenile hormone and pest control}

\section{from a Correspondent}

THE preparation of active extracts of juvenile hormone from the American cecropia silkmoth 20 years ago suggested the possibility of the eventual use of synthetic hormone as an insecticide. The original idea was that this could lead to the appearance of non-viable intermediates between larvae and adults. The discovery, 10 years later, of the chemical nature of juvenile hormones (of which three varieties have so far been described) stimulated an immense amount of synthetic chemistry in this field. A vast range of products has been produced, some of them not at all closely related chemically to the natural hormone; some, far more active than the natural substance, have been put on the market as insecticides.

The obvious theoretical objection to the juvenile hormone as an insecticide -that it will tend to produce giant larvae which may be more destructive than the ordinary sort-has proved justified in some cases. On the other hand, it has been proved empirically that some of these products are effective for rather special purposes, and some of them have a sterilising action on the adult female or disrupt the development in the resulting eggs. By and large, however, the results have been somewhat disappointing.

It was suggested early that an antijuvenile hormone, which could cause precocious metamorphosis, might be a more successful kind of insecticide. In a recent paper William $S$. Bowers and his colleagues at Geneva, New York (Science, 193, 542-547; 1976) report a search for such products in a long series of plants, by extracting the less polar organochemical components in a mixture of ether and acetone. From the common bedding out plant Ageratum houstonianum they obtained a product which had very active antijuvenile hormone properties when applied to immature Hemiptera. It in- duced precocious metamorphosis at the ensuing moult when applied to first, second or third-stage larvae of the milkweed bug Oncopeltus; and similar results were obtained in the cotton stainer Dysdercus.

The active principle was purified, analysed and synthesised. It contained two simple chromenes, 7-methoxy-2, 2-dimethyl chromene and 6,7-dimethoxy-2, 2-dimethyl chromene (Fig. 1), which were already known from plant extracts. On account of their ability to cause precocious metamorphosis these substances have been named precocene 1 and 2. Precocene 2 is about ten times more active than precocene 1 .

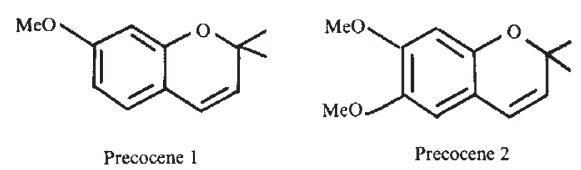

Eggs of Hemiptera fumigated with the precocenes gave rise to larvae which developed normally for two instars and then moulted to precocious adults. The precocious adults, however produced, had well formed ovaries but they never matured to form ripe eggs - a process for which juvenile hormone is necessary. Application to adult females caused regression of the ovaries; and late treatment had an ovicidal action, the nature of which is unknown.

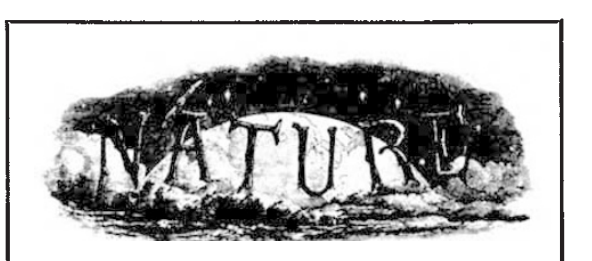

\section{A hundred years ago}

WE learn from the Chronique de l'Acclimatation, that in the just completed New York Aquarium immense basins have been constructed for the reception of the large cetaceans. A number of Otaries have already been received from Behring Strait, and the proprietors hope to be able to exhibit to the public the famous seal Ben Butler, which has for many years frequented the island of San Domingo, in the Bay of San Francisco; the director has offered 5,000 dollars for this curiosity. For the purpose of facilitating scientific researches, the central building contains a library of the best works in natural history, pictures, scientific journals, a laboratory, microscopes, drawing-tables, dissection-room, and all the necessary materials for modelling and photography. Finally, the establishment contains a restaurant in which will be served fish and crustaceans caught before the eyes of the customer.

From Nature, 14, October 5, 516; 1876.
The Colorado potato beetle Leptinotarsa, on the approach of winter, ceases to secrete juvenile hormone. It enters reproductive diapause, egg development is suppressed and it burrows into the soil. When the adult Leptinotarsa was treated with precocene it was forced, apparently permanently, into diapause. The mode of action of the precocenes is not yet known; there are many steps in the synthesis, physiological action or breakdown of the juvenile hormone, any of which could be responsible for the disruptive effects observed.

It remains to be seen whether materials of this kind will prove effective for practical control. The fact that all their effects will diminish insect damage, and that all the precocious females are sterile, are favourable factors. The substances obtained so far have induced precocious metamorphosis only in Hemiptera, and not in Holometabola. But they have sterilised adult females among Diptera and Coleoptera. The discovery of anti-juvenile hormones specific for selected pest species is an atractive possibility.

\section{Specific toxin receptors in plant disease}

\section{from I. M. Smith}

THE fungi which parasitise plants are commonly host-specific; one pathogenic species will often only infect a limited range of closely related plant species or even only some genotypes within a species. The molecular basis of this specificity is one of the outstanding problems in physiological plant pathology. Some progress has been made in the type of disease in which successful infection depends on the release by the fungus of phytotoxic metabolites which may be host specific. One notable example of this is the Victoria blight of oats (caused by Helminthosporium victoriae), in which the toxin victorin has high specific activity only on certain oat genotypes, which are at the same time the only ones susceptible to infection. Similarly, $H$. sacchari produces the toxin helminthosporoside which is only toxic to susceptible lines of sugarcane. In this case, Strobel has reported a membrane protein which binds the toxin, but only in susceptible lines. Resistant lines have a slightly different protein which does not bind. The immediate consequences of toxin binding are not clear, but this work has been till now the only reported case of a specific receptor for a phyto- 